\title{
Expected challenges of implementing universal pertussis vaccination during pregnancy in Quebec: a cross-sectional survey
}

\author{
Nicholas Brousseau MD MSc, Dominique Gagnon MSc, Maryline Vivion MA, Vanessa Poliquin MD, \\ Isabelle Boucoiran MD MSc, Bruce Tapiéro MD, Eve Dubé PhD
}

\section{Abstract}

Background: Vaccination of all pregnant women with an acellular pertussis-containing vaccine (tetanus, diphtheria, pertussis [Tdap]) was recently recommended in Canada, ideally between 27 and 32 weeks of gestation. This study aimed to describe the existing model of prenatal care in Quebec and determine to what extent maternal vaccination against pertussis could be integrated into this model.

Methods: In Quebec, health care is organized around Local Community Service Centres (LCSCs) that serve specific geographic areas. For each of 158 LCSCs ( $98.1 \%$ of LCSCs in the province), we invited 1 nurse or manager involved in prenatal care to participate in a cross-sectional Web-based survey. The structure of prenatal care visits and potential integration of maternal Tdap vaccination into the existing model were documented and compared according to urbanization level, determined with the use of census data.

Results: A completed survey was obtained for 127 LCSCs (response rate $80.4 \%$ ). Only 13 (10.2\%) and 14 (11.0\%) LCSCs offered on-site visits with a nurse for the majority of pregnant women during the second and third trimesters, respectively. A significantly higher proportion of rural LCSCs than urban LCSCs offered on-site visits to pregnant women in the third trimester $(13[18 \%]$ v. $1[2 \%])(p=0.003)$. In at least 50 LCSC service areas $(39.4 \%)$, vaccines were not available in most medical clinics offering prenatal care.

Interpretation: Given the current situation in Quebec, implementing universal maternal Tdap vaccination may be challenging, which may result in suboptimal vaccine coverage among pregnant women. As other Canadian provinces may face similar issues, a priority will be to evaluate province-based implementation models to develop efficient ways to provide maternal Tdap vaccination across Canada.

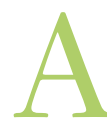

dministration of vaccines during pregnancy offers both the woman and her infant protection from certain vaccine-preventable illnesses. ${ }^{1,2}$ In Canada, the influenza vaccine has been recommended to all pregnant women since 2007. ${ }^{3}$ Recently, the National Advisory Committee on Immunization also recommended that all pregnant women receive an acellular pertussis-containing vaccine (tetanus, diphtheria, pertussis [Tdap]), ideally between 27 and 32 weeks of gestation. ${ }^{4}$ This strategy provides about $90 \%$ protection from pertussis for infants during the first 3 months of life. ${ }^{5}$ The impact of this intervention, however, hinges on uptake of the vaccine during pregnancy. In the United States, universal maternal vaccination with Tdap has been recommended since 2012, but coverage remains suboptimal., ${ }^{6,7} \mathrm{Sev}-$ eral factors contribute to low rates of vaccine coverage during pregnancy, ${ }^{8}$ but failure to make vaccination part of routine prenatal care may represent one of the main barriers. ${ }^{2,9,10}$
Numerous challenges to integrating vaccinations into routine prenatal care, including workload, costs and lack of reimbursement, have been identified in the international literature. ${ }^{11-15}$ However, given important differences in models of delivery of health care services between nations, these conclusions may not be directly applicable to the Canadian context.

Preliminary work in Quebec showed fair acceptability for maternal Tdap vaccination. ${ }^{16}$ It was also deemed important to

Competing interests: None declared.

This article has been peer reviewed.

Correspondence to: Nicholas Brousseau, nicholas.brousseau. ciussscn@ssss.gouv.qc.ca

CMAJ Open 2018. DOI:10.9778/cmajo.20180040 
understand the existing model of prenatal care and the feasibility of implementing maternal Tdap vaccination before a universal program was recommended in order to mitigate barriers that may limit vaccine coverage. ${ }^{7,10,17-19}$ In Quebec (population roughly 8.4 million; about 86000 births annually), most pregnant women initiate prenatal care in the first trimester of pregnancy, usually with a family physician (about $50 \%$ ), an obstetrician (about $45 \%$ ) or a midwife (about $5 \%$ ). Nurses at the Local Community Service Centre (LCSC) are also involved. They follow vulnerable women more actively but also routinely provide services (e.g., blood tests, prenatal classes, lactation consultant services) to other pregnant women. This model of delivering prenatal care may represent opportunities to administer vaccines during pregnancy, as the standard procedure for vaccination in Quebec is to refer to a nurse at the LCSC (pediatric and adult vaccines) or, if vaccines are available at the medical office, to vaccinate during the medical appointment (mostly adult vaccines). The objective of the present study was to better describe the model of prenatal care in Quebec and determine to what extent maternal vaccination against pertussis could be integrated into this model.

\section{Methods}

\section{Setting and participants}

In Quebec, health care is organized through 18 health regions, each of which is divided into 4-30 service areas. Each service area is served by an LCSC. To have a representative sample, we invited 1 respondent for each of 158/161 LCSCs within the province $(98.1 \%)$ to participate in this descriptive study. We excluded the 2 less populated northern health regions (Nunavik and Terres-Cries-de-la-Baie-James), which represent $1.9 \%$ of LCSCs within the province, because of important differences in the model of prenatal care delivery compared to the rest of the province. Recruitment was facilitated by regional collaborators, who sent email invitations to LCSCs in their region. Each LCSC respondent (nurse or manager) needed to have a good understanding of the model of prenatal care within the LCSC's territory. No monetary or nonmonetary incentives were offered to respondents.

\section{Measures}

We used an online survey tool (FluidSurveys [http:// fluidsurveys.com]) to administer a questionnaire on the structure of prenatal care visits both within and outside LCSCs as well as on the potential for integrating maternal vaccination into the existing prenatal model. An unpublished literature review and semistructured interviews with 11 health care professionals involved in prenatal care informed the development of the survey. The survey included 20 questions (1-4 per page, 16 pages in total) in 4 main categories: 1) prenatal care offered by the LCSC, 2) organization of antenatal ultrasonography and blood tests, 3) possible integration of vaccination services into existing model of prenatal care and 4) demographic information. We included specific questions about prenatal ultrasonography and blood tests because these activities could repre- sent opportunities within the prenatal care model to offer maternal vaccination. Most questions were closed-ended and could be answered on a Likert scale (ranging from "never" to "always"). All items included a nonresponse option when relevant, and respondents were able to change their answers through a "back" button. The questionnaire took 15-30 minutes to complete. We used adaptive questioning to reduce the number and complexity of the questions. The survey was pretested by 4 nurses in 2 different health regions to determine whether the questions would allow respondents to adequately describe the model of prenatal care in their service area and to ensure clarity; subsequently, minor adjustments were made to the survey. Data collection was carried out in November and December 2016. Participants received an email describing the study (for informed consent), with a hyperlink to the questionnaire. Two reminder emails were sent to nonrespondents.

\section{Definitions of urban and rural}

We used census data ${ }^{20,21}$ to classify LCSCs as urban or rural. Specifically, the types of dissemination areas serviced by a given LCSC determined the classification. Dissemination areas are small areas with a population of 400-700 within a census subdivision that can be categorized as being within large census metropolitan areas, other census metropolitan areas, census agglomerations or rural zones. We classified LCSCs as urban if the majority of dissemination areas within their service area were census metropolitan areas and as rural if the majority of dissemination areas within their service area corresponded to census agglomerations or rural zones.

\section{Statistical analysis}

We generated descriptive statistics for all variables. We analyzed responses to open-ended questions following standard protocols in content analysis to create categories in Excel (Microsoft). ${ }^{22} \mathrm{We}$ stratified analyses by health region in order to provide feedback on prenatal services to regional collaborators. We also performed comparisons according to urban or rural character of the LCSC service area using the Fisher exact test. The $\alpha$ level was set at $5 \%$, and tests were 2 -sided. We performed all analyses using Excel 15.0 and SAS 9.3 (SAS Institute) software.

\section{Ethics approval}

The Centre hospitalier universitaire de Québec-Université Laval Research Ethics Board reviewed the study protocol and deemed this project as program evaluation, obviating the need for approval. Only investigators had access to the data, which was stored in a secure location (password-protected access).

\section{Results}

\section{Response rate}

A completed survey was obtained for 127 of the 158 LCSCs, for a response rate of $80.4 \%$. The response rate varied by health region, from $63 \%$ to $100 \%$; at least 4 LCSCs from every health region were represented. Table 1 summarizes the response rate by health region and by the respondents' 


\begin{tabular}{|c|c|c|c|c|c|}
\hline \multirow[b]{3}{*}{$\begin{array}{l}\text { Health } \\
\text { region }\end{array}$} & \multicolumn{5}{|c|}{ No. $(\%)$ of LCSCs } \\
\hline & \multirow[b]{2}{*}{ Overall } & \multicolumn{2}{|c|}{ Profession } & \multicolumn{2}{|c|}{ Urban v. rural } \\
\hline & & Nurses & Managers & $\begin{array}{l}\text { Within urban } \\
\text { LCSC service } \\
\text { area }\end{array}$ & $\begin{array}{l}\text { Within rural } \\
\text { LCSC service } \\
\text { area }\end{array}$ \\
\hline A & $5 / 8(62)$ & $4 / 5(80)$ & $1 / 5(20)$ & $0 / 5(0)$ & $5 / 5(100)$ \\
\hline B & 6/6 (100) & $3 / 6(50)$ & $3 / 6(50)$ & $3 / 6(50)$ & $3 / 6(50)$ \\
\hline C & 10/13 (77) & $10 / 10(100)$ & $0 / 10(0)$ & $5 / 10(50)$ & $5 / 10(50)$ \\
\hline D & $8 / 12(67)$ & $8 / 8(100)$ & $0 / 8(0)$ & $1 / 8(12)$ & 7/8 (88) \\
\hline$E$ & 9/9 (100) & $7 / 9(78)$ & 2/9 (22) & $1 / 9(11)$ & 8/9 (89) \\
\hline $\mathrm{F}$ & 23/30 (77) & $13 / 23(56)$ & $10 / 23(43)$ & 23/23 (100) & $0 / 23(0)$ \\
\hline G & 7/8 (88) & 4/7 (57) & $3 / 7(43)$ & $4 / 7(57)$ & $3 / 7(43)$ \\
\hline $\mathrm{H}$ & $4 / 6(67)$ & $4 / 4(100)$ & $0 / 4(0)$ & $0 / 4(0)$ & 4/4 (100) \\
\hline I & 6/9 (67) & 5/6 (83) & $1 / 6(17)$ & $0 / 6(0)$ & $6 / 6(100)$ \\
\hline J & 4/4 (100) & $4 / 4(100)$ & $0 / 4(0)$ & $0 / 4(0)$ & $4 / 4(100)$ \\
\hline K & 6/8 (75) & 6/6 (100) & $0 / 6(0)$ & $0 / 6(0)$ & $6 / 6(100)$ \\
\hline L & 7/11 (64) & 7/7 (100) & $0 / 7(0)$ & $1 / 7(14)$ & 6/7 (86) \\
\hline M & $4 / 4(100)$ & $4 / 4(100)$ & $0 / 4(0)$ & $4 / 4(100)$ & $0 / 4(0)$ \\
\hline $\mathrm{N}$ & $6 / 6(100)$ & 2/6 (33) & $4 / 6(67)$ & 2/6 (33) & $4 / 6(67)$ \\
\hline $\mathrm{O}$ & 6/7 (86) & $6 / 6(100)$ & $0 / 6(0)$ & $3 / 6(50)$ & $3 / 6(50)$ \\
\hline$P$ & $16 / 17(94)$ & $8 / 16(50)$ & $8 / 16(50)$ & $9 / 16(56)$ & $7 / 16(44)$ \\
\hline Total & 127/158 (80.4) & $95 / 127(74.8)$ & $32 / 127$ (25.2) & $56 / 127(44.1)$ & $71 / 127(55.9)$ \\
\hline
\end{tabular}

profession and urban/rural classification. Of the 127 LCSCs, $56(44.1 \%)$ were classified as urban and 71 (55.9\%) as rural. The proportion classified as urban was similar for participating and nonparticipating (48\%) LCSCs.

\section{Prenatal care offered by Local Community Service Centres}

New pregnancies were routinely notified to more than half (69 [54.3\%]) of LCSCs, usually by family physicians and obstetricians after the first medical visit. A standardized form was used in most (63 [91\%]) of these LCSC service areas. This voluntary mechanism provided the LCSC with contact information for pregnant women in their service area in order to offer them prenatal care. A significantly higher proportion of rural LCSCs than urban LCSCs were informed of new pregnancies $(54$ [76\%] v. $15[27 \%])(p<0.001)$.

Few LCSCs offered on-site visits to pregnant women (Figure 1). During the third trimester, only 14 LCSCs (11.0\%) offered on-site visits with a nurse for a majority of pregnant women. A significantly higher proportion of rural LCSCs than urban LCSCs offered on-site visits to pregnant women (first trimester: 24 [34\%] v. 6 [11\%], $p=0.003$; second trimester: $13[18 \%]$ v. $0[0 \%], p<0.001)$; third trimester: 13 $[18 \%]$ v. $1[2 \%], p=0.003)$.

\section{Organization of prenatal ultrasonography and blood tests}

Most respondents (118 [92.9\%]) indicated that secondtrimester prenatal ultrasonography was conducted at the hospital in most cases. First-trimester blood tests (e.g., complete blood count and serologic testing) were offered mainly in hospitals for 71 service areas (55.9\%) and at the LCSC for 54 service areas $(42.5 \%)$. Findings were similar for second-trimester blood tests, which included the oral glucose challenge test (Figure 2). When blood tests were performed at the LCSC, most blood samples were drawn by a medical laboratory technologist, not a nurse.

\section{Integration of vaccination services into existing health care visits}

According to 50 respondents (39.4\%), vaccines were available in a minority $(25[19.7 \%])$ or none $(25$ [19.7\%]) of the medical clinics offering prenatal care within their LCSC service area. This situation may be even more common, as a substantial number of respondents (54 [42.5\%]) could not answer this question. Only 23 respondents $(18.1 \%)$ mentioned that vaccines were available in most medical clinics offering prenatal care within their LCSC service area. Availability of vaccines also depended on the urbanization level: vaccines were more 


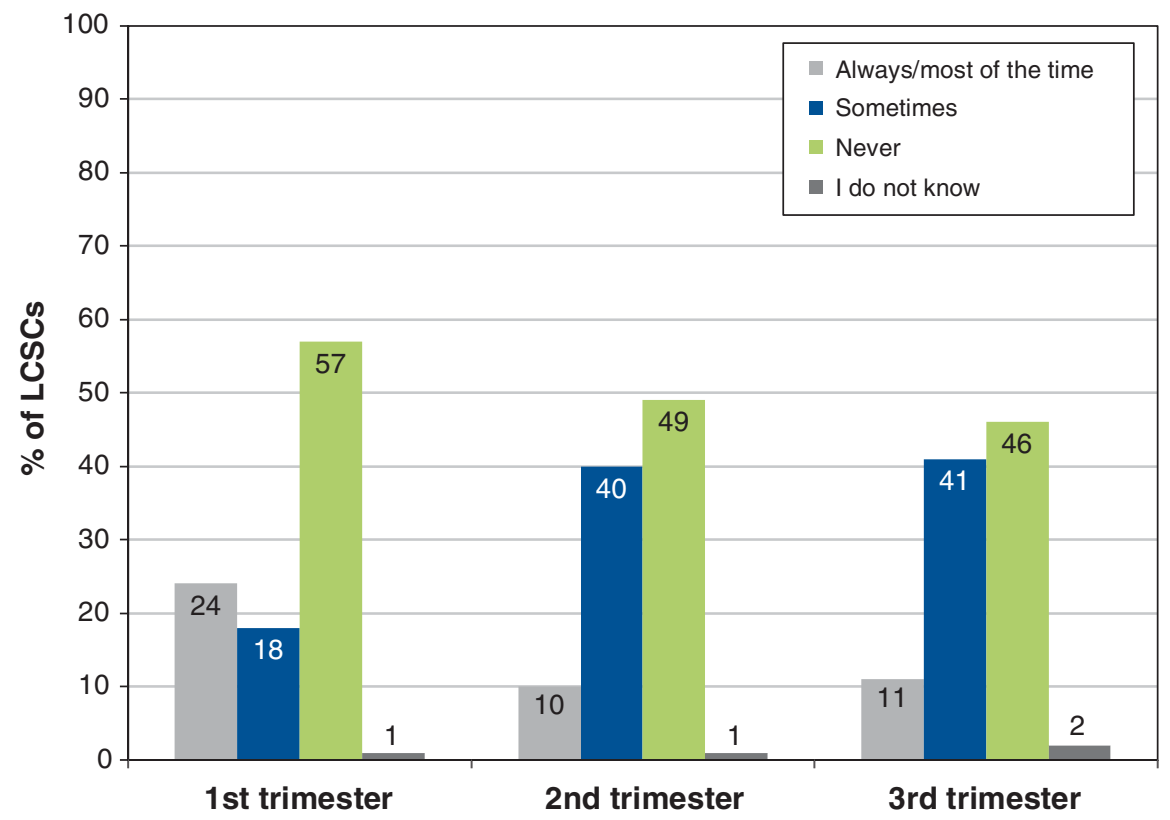

Figure 1: Proportion of Quebec Local Community Service Centres (LCSCs) offering on-site visits with a nurse, by trimester. Always/ most of the time $=$ for the majority of pregnant women from their service area, sometimes $=$ for a minority of pregnant women from their service area, never = for none or almost none of pregnant women from their service area. $n=127$ for each trimester.

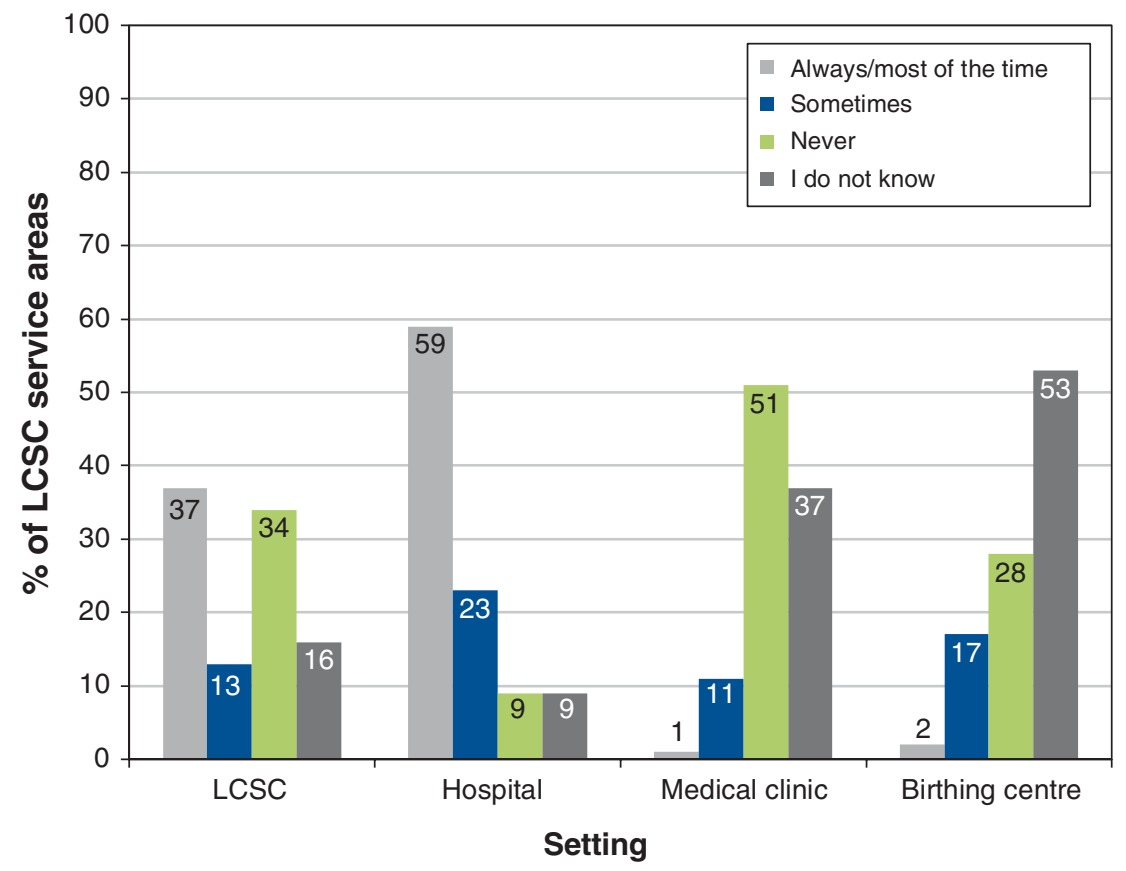

Figure 2: Reported settings in which second-trimester blood tests (including the oral glucose challenge test) were offered. Always/most of the time $=$ for the majority of pregnant women from their service area, sometimes $=$ for a minority of pregnant women from their service area, never = for none or almost none of pregnant women from their service area. Note: LCSC = Local Community Service Centre. 
frequently unavailable in medical clinics offering prenatal care in rural LCSC service areas than in urban LCSC service areas (22 [31\%] v. $3[5 \%])(p<0.001)$.

Participants also gave their opinion on the best way to reach pregnant women for systematic vaccination. Responses differed significantly according to urbanization level (Figure 3). Most respondents (48 [86\%]) from urban LCSCs stated that medical clinics should be chosen "always" or "often" for vaccination of pregnant women, compared to 24 (34\%) of those from rural LCSCs $(p<0.001)$. The majority (43 [61\%]) of respondents from rural LCSCs preferred vaccination within LCSCs by nurses offering prenatal care, compared to 10 respondents (18\%) from urban LCSCs $(p<0.001)$.

\section{Interpretation}

Our study shows that, currently in Quebec, rural LCSCs are more involved in prenatal care than urban LCSCs and may be well suited to offer vaccination during pregnancy. However, even in rural LCSCs, only a minority of pregnant women are seen on-site during the second and third trimesters, when the administration of Tdap is recommended. ${ }^{4}$ Only 1 in 10 LCSCs would be able to integrate vaccination into the existing prenatal care model without scheduling an additional visit. An alternative model of vaccine delivery would be through medical clinics offering prenatal care. However, our results suggest that a high proportion of such medical clinics may not stock vaccines. As such, the province may face important challenges when integrating universal maternal Tdap vaccination into the existing model of prenatal care. As ease of access to vaccination services is an important determinant of uptake, the need to schedule additional appointments for administration of the Tdap vaccine may result in suboptimal vaccine coverage among pregnant women. ${ }^{23}$ It may also be necessary to invest considerable resources in order to deliver Tdap vaccination during pregnancy, which would affect the cost-effectiveness of the program.

Other similarly resourced countries, including the US and the United Kingdom, have recommended universal Tdap vaccination during pregnancy for several years. The reported coverage rates in these countries vary considerably. In the US, coverage rates for Tdap vaccination during pregnancy are slowly improving and approximate $50 \%,{ }^{24-29}$ but implementation has been reported to be challenging. ${ }^{6,30}$ In countries such as the UK that have achieved higher coverage (about 70\%) for Tdap vaccination during pregnancy, ${ }^{31}$ vaccines are commonly administered in the physician's office. ${ }^{9,32}$ It is important to note that many pregnant women in the UK receive care within the context of a general medical clinic that stocks vaccines. This suggests that the ability to vaccinate at the point of prenatal care may facilitate implementation of universal maternal vaccination. ${ }^{5,33}$ Currently in Quebec, there are barriers to implementation of maternal vaccination in a similar manner as in the UK. First, most medical clinics delivering prenatal care do not appear to stock vaccines and are not the usual vaccination providers. Therefore, if vaccination of pregnant women is expected to be provided in medical clinics, this strategy will require

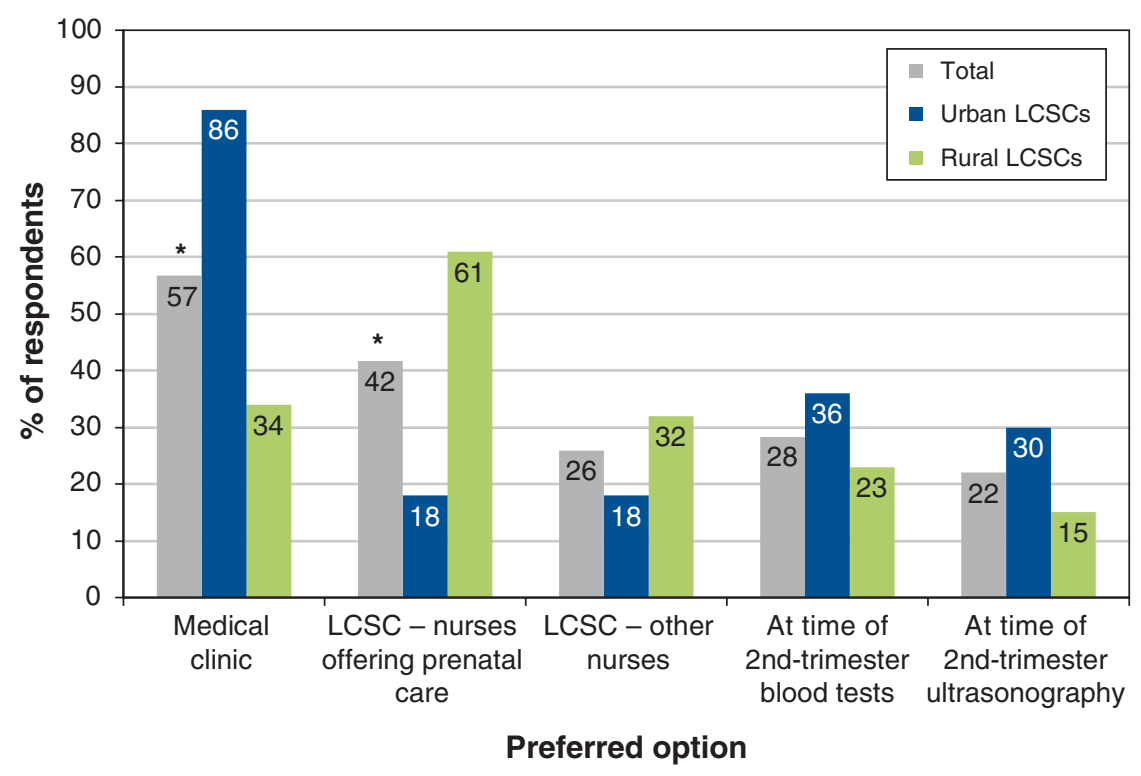

Figure 3: Respondents' preferred options for tetanus, diphtheria, pertussis (Tdap) vaccine administration if universal maternal Tdap vaccination were to be implemented. Proportions correspond to the proportion of respondents who indicated that a given option would be chosen "always" or "often." Respondents could select more than 1 option. *Results significantly different between urban and rural Local Community Service Centres (LCSCs) $(p<0.05)$. 
special training as well as infrastructure for vaccine storage and handling. Furthermore, physicians in Quebec do not receive any financial compensation for administering vaccines. ${ }^{34}$ Financial barriers such as inadequate reimbursement have been described in other countries. ${ }^{9,24}$

After interviewing obstetricians, general practitioners and midwives, Webb and colleagues ${ }^{10}$ concluded that "the incorporation of maternal immunization into standard care through a structured process is an important facilitator for immunization uptake." Certainly, there are a number of instances during prenatal care in the Canadian context that may represent ideal clinical encounters to integrate counselling and delivery of maternal vaccination. Anecdotally, we have found that many prenatal care providers consider the 26to 28-week visit for the oral glucose challenge test an ideal moment for counselling and administration of the Tdap vaccine given that is it recommended to the vast majority of pregnant women and that patients are already planning for a longer visit because of the length of the test. However, our results indicate that fewer than half of oral glucose challenge tests are performed at LCSCs, which suggests that simple integration of Tdap vaccination at these venues would not cover all pregnant women in Quebec. Additional work is needed to evaluate these potential strategies by documenting costs, facilitators, barriers and vaccine uptake obtained.

\section{Limitations}

Although we targeted nearly all LCSCs in the province and obtained a good response rate $(80 \%)$, there are some limitations to the generalizability of our findings. First, because our survey was limited to Quebec, our results may not be representative of challenges that other Canadian jurisdictions may face given that the organization of prenatal health care services varies greatly between jurisdictions. Furthermore, the respondents were frequently not able to answer questions about services outside the LCSC (e.g., availability of vaccines in medical offices), which may have led to information bias. However, preliminary results from a survey targeting physicians throughout Canada suggest that, for several provinces, a substantial proportion of physicians offering prenatal care do not administer vaccines. ${ }^{35}$ Finally, we relied on the response of 1 representative from each LCSC, and we cannot rule out the possibility that respondents' answers may not perfectly reflect the situation in the LCSC service area.

\section{Conclusion}

Implementation of a recommendation to offer universal maternal Tdap vaccination may be challenging in Quebec, which may result in suboptimal vaccine coverage among pregnant women. This study did not identify a unique model of integrating Tdap vaccination through the existing care structures at LCSCs, and the findings suggest that there may also be barriers to implementation through medical clinics. Other Canadian provinces may face similar challenges. It will be important to evaluate province-based implementation models to develop efficient ways to provide maternal Tdap vaccination across Canada. The groundwork for integrating
Tdap vaccination into the existing model of prenatal care may also facilitate the implementation of maternal vaccination for other pathogens (e.g., group B Streptococcus) in the future.

\section{References}

1. Baxter R, Bartlett J, Fireman B, et al. Effectiveness of vaccination during pregnancy to prevent infant pertussis. Pediatrics 2017;139:e20164091.

2. Madhi SA, Cutland CL, Kuwanda L, et al. Influenza vaccination of pregnant women and protection of their infants. NEngl f Med 2014;371:918-31.

3. National Advisory Committee on Immunization (NACI). Statement on influenza vaccination for the 2007-2008 season. Can Commun Dis Rep 2007; 33(ACS-7):1-38

4. Update on immunization in pregnancy with tetanus, toxoid, reduced diphtheria toxoid and reduced acellular pertussis (Tdap) vaccine. Ottawa: Public Health Agency of Canada and National Advisory Committee on Immunization; 2018.

5. Amirthalingam G, Campbell H, Ribeiro S, et al. Sustained effectiveness of the Maternal Pertussis Immunization Program in England 3 years following introduction. Clin Infect Dis 2016;63(Suppl 4):S236-43.

6. Ahluwalia IB, Ding H, D'Angelo D, et al. Tetanus, diphtheria, pertussis vaccination coverage before, during, and after pregnancy - 16 states and New York City, 2011. MMWR Morb Mortal Wkly Rep 2015;64:522-6.

7. Krishnaswamy S, Wallace E, Buttery J, et al. Antenatal pertussis vaccination: Are we implementing best evidence into practice? Aust N Z 7 Obstet Gynaecol 2016;56:552-5.

8. Wilson RJ, Paterson P, Jarrett C, et al. Understanding factors influencing vaccination acceptance during pregnancy globally: a literature review. Vaccine 2015; 33:6420-9.

9. The National Vaccine Advisory Committee: reducing patient and provider barriers to maternal immunizations. Public Health Rep 2015;130:10-42.

10. Webb H, Street J, Marshall H. Incorporating immunizations into routine obstetric care to facilitate health care practitioners in implementing maternal immunization recommendations. Hum Vaccin Immunother 2014;10:1114-21.

11. Bonville CA, Cibula DA, Domachowske JB, et al. Vaccine attitudes and practices among obstetric providers in New York State following the recommendation for pertussis vaccination during pregnancy. Hum Vaccin Immunother 2015; 11:713-8.

12. Jones KM, Carroll S, Hawks D, et al. Efforts to improve immunization coverage during pregnancy among ob-gyns. Infect Dis Obstet Gynecol 2016;2016: 6120701 .

13. Kissin DM, Power ML, Kahn EB, et al. Attitudes and practices of obstetriciangynecologists regarding influenza vaccination in pregnancy. Obstet Gynecol 2011;118:1074-80.

14. Leddy MA, Anderson BL, Power ML, et al. Changes in and current status of obstetrician-gynecologists' knowledge, attitudes, and practice regarding immunization. Obstet Gynecol Surv 2009;64:823-9.

15. Power ML, Leddy MA, Anderson BL, et al. Obstetrician-gynecologists' practices and perceived knowledge regarding immunization. Am 7 Prev Med 2009 37:231-4.

16. Vivion M, Gagnon D, Dubé E, et al. Vaccination en cours de grossesse : acceptabilité pour les femmes enceintes et enjeux de faisabilité. Québec: Direction des risques biologiques et de la santé au travail, Institut national de santé publique du Québec; 2017.

17. Donaldson B, Jain P, Holder BS, et al. What determines uptake of pertussis vaccine in pregnancy? A cross sectional survey in an ethnically diverse population of pregnant women in London. Vaccine 2015;33:5822-8.

18. Tong A, Biringer A, Ofner-Agostini M, et al. A cross-sectional study of maternity care providers' and women's knowledge, attitudes, and behaviours towards influenza vaccination during pregnancy. 7 Obst Gynaecol Can 2008;30:404-10.

19. Vilca ML, Esposito $S$. The crucial role of maternal care providers as vaccinators for pregnant women. Vaccine 2018;36:5379-84.

20. Indice de défavorisation, Canada, 2011. Québec: Institut national de santé publique du Québec; 2011. Available: https://www.inspq.qc.ca/expertises/ gestion-et-analyse-de-l-information/diffusion-d-une-information-adaptee/ santescope/indice-de-defavorisation/indice-de-defavorisation-canada-2011 (accessed 2017 Aug. 14).

21. Statistical Area Classification (SAC). Ottawa: Statistics Canada; 2015. Cat. no. 92-195-X. Available: https://www150.statcan.gc.ca/n1/pub/92-195-X/2011001/ other-autre/sac-css/sac-css-eng.htm (accessed 2017 Aug. 14).

22. Hsieh HF, Shannon SE. Three approaches to qualitative content analysis. Qual Health Res 2005; 15:1277-88.

23. Centers for Disease Control and Prevention (CDC). Influenza vaccination coverage among pregnant women: 2011-12 influenza season, United States. MMWR Morb Mortal Wkly Rep 2012;61:758-63.

24. Harriman K, Winter K. Pertussis vaccine uptake during pregnancy: We need to do better in the US. Prev Med 2014;67:320-1.

25. Healy CM, Ng N, Taylor RS, et al. Tetanus and diphtheria toxoids and acellular pertussis vaccine uptake during pregnancy in a metropolitan tertiary care center. Vaccine 2015;33:4983-7. 
26. Kerr S, Van Bennekom CM, Liang JL, et al. Tdap vaccination coverage during pregnancy - selected sites, United States, 2006-2015. MMWR Morb Mortal Wkly Rep 2017;66:1105-8.

27. Kharbanda EO, Vazquez-Benitez G, Lipkind HS, et al. Maternal Tdap vaccination: coverage and acute safety outcomes in the vaccine safety datalink, 2007-2013. Vaccine 2016;34:968-73.

28. Koepke R, Kahn D, Petit AB, et al. Pertussis and influenza vaccination among insured pregnant women - Wisconsin, 2013-2014. MMWR Morb Mortal Wkly Rep 2015;64:746-50.

29. Koerner J, Forinash AB, Yancey AM, et al. Administration rates of the Tdap vaccine in obstetric patients. Ann Pharmacother 2018;52:655-61.

30. Housey M, Zhang F, Miller C, et al. Vaccination with tetanus, diphtheria, and acellular pertussis vaccine of pregnant women enrolled in Medicaid - Michigan, 2011-2013. MMWR Morb Mortal Wkly Rep 2014;63:839-42.

31. Pertussis vaccination programme for pregnant women update: vaccine coverage in England, July to September 2017. Wellington (UK): Public Health England; 2018.

32. Maertens K, Hoang TT, Nguyen TD, et al. The effect of maternal pertussis immunization on infant vaccine responses to a booster pertussis-containing vaccine in Vietnam. Clin Infect Dis 2016;63(Suppl 4):S197-204.

33. Cherry JD. Tetanus-diphtheria-pertussis immunization in pregnant women and the prevention of pertussis in young infants. Clin Infect Dis 2015;60:338-40.

34. Abolition des frais accessoires - le règlement en vigueur dès le 26 janvier 2017 [press release]. Québec: Ministère de la Santé et des Services sociaux; 2017 Jan. 11.

35. Cook J, Dubé E, Kaminsky K, et al. Vaccination of pregnant women: Are maternity care providers hesitant? [poster]. Society of Obstetricians and Gynaecologists of Canada/Association of Academic Professionals in Obstetrics and Gynaecology Ontario CME 2017; 2017 Nov. 30-Dec. 2; Toronto.
Affiliations: Institut national de santé publique du Québec (Brousseau, Gagnon, Vivion, Dubé); Université Laval (Brousseau, Vivion, Dubé), Québec, Que.; Department of Obstetrics, Gynecology and Reproductive Sciences (Poliquin), University of Manitoba, Winnipeg, Man.; Centre hospitalier universitaire Sainte-Justine (Boucoiran, Tapiéro), Montréal, Que.

Contributors: Nicholas Brousseau conceived the study and coordinated the analysis. Dominique Gagnon, Maryline Vivion, Bruce Tapiéro and Eve Dubé contributed to the study design. All of the authors contributed to data analysis and interpretation, and drafting of the manuscript, critically revised the manuscript for important intellectual content, gave final approval of the version to be published and agreed to be accountable for all aspects of the work

Funding: This work was supported by the Quebec Ministry of Health and Social Services.

Acknowledgements: The authors thank Caroline Boudreault-Fiset, Gaël Chetaille, Marie-France Richard and Josiane Rivard for their contribution to data collection and analysis. They also thank François Boucher and Monique Landry for their contribution to the study design. Finally, they thank all the regional collaborators and Local Community Service Centre respondents who made this project possible.

Supplemental information: For reviewer comments and the original submission of this manuscript, please see www.cmajopen.ca/content/6/3/ E391/suppl/DC1. 